\title{
EFEITOS DE FONTES E DOSES DE SILICATO DE CÁLCIO NO RENDIMENTOAGRÍCOLA E NA QUALIDADE TECNOLÓGICA DA CANA-DE-AÇÚCAR, CULTIVAR SP80-1816 ${ }^{1}$
}

\author{
Effects of sources and dosages of calcium silicate on yeld and quality \\ of sugarcane, cultivar SP80-1816
}

\author{
Gustavo Melasipo Vilela Leite ${ }^{2}$, Luiz Antônio de Bastos Andrade ${ }^{3}$ Júlio César Garcia ${ }^{4}$, Ivan Antônio dos Anjos ${ }^{4}$
}

\begin{abstract}
RESUMO
Com o objetivo de estudar os efeitos da aplicação de diferentes fontes e doses de silicato de cálcio na cultura da cana-deaçúcar, cultivar SP80-1816, instalou-se um experimento num Latossolo Vermelho Amarelo distrófico, em área localizada no alambique João Mendes - "JM" (Sítio Vó Zirica), no Município de Perdões, Estado de Minas Gerais. O delineamento experimental utilizado foi o de blocos ao acaso, quatro repetições, esquema fatorial 3x3+1, sendo três fontes de silicato de cálcio (Silifértil, Extrativa e Agronelli) e três doses $(150,300$ e $450 \mathrm{~kg} / \mathrm{ha}$ ), mais um tratamento adicional, sem silicato(controle), totalizando 10 tratamentos. As diferentes fontes e doses foram aplicadas no sulco de plantio juntamente com adubação NPK. A aplicação de silicato de cálcio no sulco de plantio, independente das fontes e doses utilizadas, proporcionou um aumento no teor de fibra (\%) cana, mas não influenciou o rendimentos de colmos colhidos e de açúcar total recuperável (ATR), além dos teores de brix, pol e pureza $(\%)$ cana.
\end{abstract}

Termos para indexação: Cana-de-açúcar, adubação, silicato de cálcio.

\section{ABSTRACT}

With the objective of studying the effects of the application of different sources and doses of calcium silicate on the sugarcane culture, cultivar SP80-1816, one installed an experiment in a Typic Hapludox, in an area located at the João Mendes-"JM" distillery (Sítio Vó Zirica), in the Municipal district of Perdões, in the state of Minas Gerais. A random block experimental layout was used, with four repetitions and a $3 \times 3+1$ factorial outline, three being sources of calcium silicate (Silifértil, Extrativa e Agronelli) and three being doses (150, 300 and $450 \mathrm{~kg} / \mathrm{ha}$ ), an additional treatment, without silicate (control), totalizing 10 treatments. The different sources and doses were applied in the planting furrow together with NPK manuring. The application of calcium silicate in the planting furrow, independently of the sources and doses used, provided an increase in the fiber level percent/cane, but didn't influence the total yield recoverable sugar (ATR) brix, pol e pureness (\%) of cane plant.

Index terms: Sugarne, manuring, calcium silicate.

(Recebido em 26 de abril de 2006 e aprovado em 9 de maio de 2007)

\section{INTRODUÇÃO}

A cana-de-açúcar ocupa papel de destaque no cenário econômico e social, principalmente na geração de divisas, na expansão de novas fronteiras agrícolas e na utilização intensiva de mão-de-obra direta e indireta. Segundo a primeira estimativa do governo brasileiro realizada pela Companhia Nacional de Abastecimento (AGRIANUAL, 2005), a colheita da safra 2005/06 deverá atingir 450,2 milhões de toneladas de colmos.

Deste total, 379,2 milhões de toneladas seriam do Centro sul do país, responsável por $85 \%$ da produção nacional, e 71 milhões de toneladas serão colhidas no
Nordeste do país. A área total cultivada está estimada em 6,09 milhões de hectares.

Ainda segundo o Agrianual (2005), as unidades produtoras do Brasil deverão destinar 401,5 milhões de toneladas de colmos para produzir açúcar e álcool. A produção de açúcar está estimada em 27,2 milhões de toneladas, absorvendo 218,7 milhões de toneladas de cana. A produção de álcool - estimada em 17,5 bilhões de litros - deverá absorver 182,8 milhões de toneladas de cana, e o volume restante $-48,7$ milhões de toneladas - serão destinados à cachaça, rapadura e ração animal.

Estes números elevados, tanto na área plantada como em produção, tiveram início com a implantação do

\footnotetext{
${ }^{1}$ Parte da Dissertação apresentada á Universidade Federal de Lavras/UFLA-Caixa Postal 3037-37200-000- Lavras -MG, pelo primeiro autor, como um dos requisitos do curso de mestrado em agronomia, área de concentração Fitotecnia-apoio financeiro CAPES

Engenheiro Agrônomo, Mestre - Gerência Agrícola - Usina Luciânia - Zona Rural - Lagoa da Prata, MG - 35590-000 - gustavilela@gmail.com ${ }^{3}$ Doutor - Departamento de Agricultura/DAG - Universidade Federal de Lavras/UFLA - Cx. P. 3037 - 37200-000 - Lavras, MG - laba@ufla.br ${ }^{4}$ Engenheiros Agrônomos, Doutores - Centro Avançado de Pesquisa Tecnológica do Agronegócio de Cana/APTA/IAC - Anel Viário Contorno Sul, Km 321 Cx. P. 306 - 14001-970 - Ribeirão Preto, SP - jcgarcia@iac.sp.gov.br; iaanjos@iac.sp.gov.br
} 
Programa Nacional do Álcool (PROÁLCOOL) em 1975, que resultou na expansão da área cultivada com cana-de-açúcar para áreas marginais, ou seja, solos com menor fertilidade. A baixa disponibilidade de nutrientes, baixa capacidade de troca catiônica, elevada acidez e altos níveis de alumínio e manganês, são em geral características de solos de baixa fertilidade. Com isso passou-se a necessitar de maior tecnificação na área de nutrição mineral de plantas e fertilidade do solo para a cultura da cana-de-açúcar. Neste contexto, pode-se incluir a adubação com silício como uma das fontes alternativas, objetivando aumento de produtividade, uma vez que a extração deste elemento pela cultura da cana-de-açúcar é muito alto, com níveis próximos a extração de "N"(MANECHINI \& DONZELLI, 1984), além de proporcionar resistência a pragas e doenças de acordo com (KORNDÖRFER, 1996).

As escórias de siderurgia podem ser utilizadas na agricultura basicamente como fornecedoras de $\mathrm{Ca}, \mathrm{Mg}$ e Si para as culturas ou como corretivo da acidez do solo. Entretanto, estes materiais, apesar de estarem disponíveis no mercado brasileiro, têm sido pouco comercializados para esse fim (QUAGGIO, 2000).

Com a aplicação da escória, ocorre normalmente a correção da acidez do solo, tendo em vista a ação do silicato de cálcio, devido à base química $\mathrm{SiO}_{3}{ }^{-2}$, ocorrendo, desta forma, algumas reações químicas no solo de maneira semelhante ao calcário, como aumento do $\mathrm{pH}$ e precipitação de Al e Mn tóxicos (PRADO \& FERNANDES, 2001).

O elemento silício $(\mathrm{Si})$ tem recebido pouca atenção dos pesquisadores, provavelmente pelo fato de não estar incluído no grupo de elementos considerados como essenciais para as plantas. No entanto, os efeitos benéficos do Si têm sido demonstrados em várias espécies vegetais, especialmente quando estas plantas são submetidas a algum tipo de estresse, seja ele de caráter biótico ou abiótico (DATNOFF et al., 2001; FARIA, 2000; KORNDÖRFER et al., 2002).

Gramíneas em geral, dentre elas a cana-de-açúcar, são classificadas como acumuladoras de Si e os depósitos deste elemento são encontrados nas paredes celulares, no lúmen celular e em localizações extracelulares (YOSHIDA, 1965).

O "Si" aparece na cana-de-açúcar em altas concentrações, podendo variar desde $0,14 \%$ em folhas jovens até $6,7 \%$ nos colmos e folhas velhas. No Havaí, as folhas contendo menos de $0,5 \%$ de "Si" são freqüentemente afetadas por um sintoma denominado "freckling". A causa deste sintoma é ainda bastante controvertida, porém a maioria dos pesquisadores o atribuem a falta de "Si" e a desequilíbrios nutricionais. $\mathrm{O}$ aparecimento da Ferrugem na cana-de-açúcar (Puccinia melanocephala) pode estar relacionado ao referido sintoma. $\mathrm{O}$ sintoma é mais severo nas folhas velhas e a área fotossintética é normalmente fortemente atingida. De acordo com Fox et al. (1967), a aplicação de 6 t/ha, de silicato ao solo resolve o problema.

Cheong et al. (1973) concluíram que o aparecimento de "sarda" nas folhas de cana-de-açúcar se deveu à deficiência de "Si" e não à toxidez de ferro ou manganês como era atribuído. Segundo ainda o mesmo autor, o "Si" se acumula nos espaços interfibrilares, reduzindo o movimento da água através da parede celular, aumentando assim a economia de água da planta pela diminuição da taxa de transpiração.

Anderson et al. (1987), obtiveram um aumento na produtividade de colmos de cana-de-açúcar de $39 \%$ e de $50 \%$ na produção de açúcar, com a aplicação de 20 toneladas de escórias silicatadas por hectare no plantio.

Para Kidder \& Gascho (1977), os solos carentes em "Si" resultam na disseminação rápida do mosaico, atraso na maturação e diminuição da quantidade de sólidos solúveis no caldo de cana. Mas é na menor produtividade de colmos o principal reflexo da deficiência do "Si" na cana-de-açúcar. Ainda segundo os mesmos autores, os aumentos de produtividades de cana-de-açúcar variaram entre 10 e $35 \%$ com aplicação de silicato no solo, não havendo efeitos nos teores de sacarose.

Prado (2000), avaliando a resposta da cana-deaçúcar à aplicação de escória silicatada como corretivo de acidez do solo, observou que o perfilhamento da cana-deaçúcar foi influenciado positivamente pela aplicação da escória de siderurgia, na cana-planta e cana-soca, alcançando a produtividade de 100 e $75 \mathrm{t} \mathrm{ha}^{-1}$, respectivamente, superiores às obtidas com a testemunha $\left(\right.$ cana-planta $=89$ e cana-soca $\left.=58 \mathrm{t} \mathrm{ha}^{-1}\right)$. Prado \& Fernandez (2001), em um experimento conduzido em condições de campo, em um Latossolo Vermelho-Amarelo distrófico com cana-de-açúcar (SP80-1842), compararam a escória de siderurgia (aciaria) e um calcário calcítico acrescido com micronutrientes, equilibrando-se com o da escória, ambos aplicados em doses equivalentes a $\mathrm{CaCO}_{3}$ iguais a 1,3; 3,6 e 7,5 $\mathrm{t} \mathrm{ha}^{-1}$. Essas doses tinham por objetivo elevar a saturação por bases do solo a 50; 75 e 100\%. Para o rendimento de colmos e para o perfilhamento, a resposta da cana-de-açúcar (média de dois cortes) foi quadrática e linear em função da aplicação do calcário e da escória, respectivamente. Concluiram que o maior perfilhamento, resultante da aplicação da escória de siderurgia, contribuiu para o maior rendimento de colmos da cana-de-açúcar.

Silveira Júnior et al. (2003) também obtiveram aumentos de produtividade de colmos na cana-planta e 
cana-soca com o uso de silicato, sendo estes aumentos superiores aos efeitos do calcário.

As escórias de siderurgia que contêm silício em suas composições químicas, são um passivo para a indústria do ferro. Estudos que venham a comprovar sua utilidade na agricultura, tanto como corretivo de solo, como fonte de silício, podem viabilizar sua utilização em escala comercial.

O Brasil é o sexto maior produtor mundial de ferrogusa com produção anual de cerca de 25 milhões de toneladas, gerando por volta de 6,25 milhões de toneladas de escória anualmente, sendo o Estado de Minas Gerais o maior produtor nacional, responsável por mais da metade de todo o ferro-gusa e aço produzidos internamente (KORNDÖRFER et al., 2002).

Um problema seria os locais de produção das escórias, muitas das vezes distantes dos locais de utilização, encarecendo o custo do produto em função do frete. A aplicação concentrada deste produto no sulco de plantio poderia viabilizar sua utilização em locais mais distantes, contribuindo ainda para reduzir o passivo ambiental.

O objetivo deste trabalho foi o de estudar os efeitos de fontes e doses de silicato de cálcio concentradas no sulco de plantio, no rendimento agrícola e na qualidade tecnológica da cana-de-açúcar, cultivar SP80-1816.

\section{MATERIALE MÉTODOS}

O experimento foi conduzido em área localizada no alambique João Mendes - "JM" (Sítio Vó Zirica), no Município de Perdões, situado na região Campos das vertentes do Estado de Minas Gerais, cujas coordenadas geográficas são latitude de $21^{\circ} 05^{\prime} 20$, longitude de $45^{\circ} 05^{\prime}$ 50 "e altitude de 826 metros.

O clima da região, segundo a classificação de Köppen, é do tipo Cwb, caracterizado por uma estação seca entre abril e setembro e uma estação chuvosa de outubro a março. A região apresenta uma média anual de precipitação pluvial e temperatura de 1493,2 mm e 19,3 C, respectivamente (BRASIL, 1992).

O solo no qual foi instalado o experimento caracteriza-se como Latossolo Vermelho Amarelo distrófico, com relevo plano à suave ondulado.

No mês de setembro de 2003, foram retiradas amostras de solo, profundidades de $0-20 \mathrm{~cm}$ e $20-40 \mathrm{~cm}$, para determinação das características químicas do solo, realizadas no Laboratório de Análise de Solos da Universidade Federal de Lavras (UFLA), Lavras -MG. Os resultados das análises química do solo encontram-se na Tabela 1.
Tabela 1 - Características químicas do solo da área experimental, profundidades de $0-20 \mathrm{~cm}$ e de $20-40 \mathrm{~cm}$. UFLA, Lavras-MG, 2004

\begin{tabular}{|c|c|c|c|}
\hline \multicolumn{2}{|c|}{ Atributo } & \multicolumn{2}{|c|}{ Profundidades } \\
\hline & & $0-20 \mathrm{~cm}$ & $20-40 \mathrm{~cm}$ \\
\hline $\mathrm{Al}$ & cmol..dm ${ }^{-3}$ & 0,4 & 0,4 \\
\hline $\mathrm{Ca}$ & cmol..dm ${ }^{-3}$ & 1,7 & 0,7 \\
\hline $\mathrm{Mg}$ & cmol..dm ${ }^{-3}$ & 0,4 & 0,2 \\
\hline K & mg...dm ${ }^{-3}$ & 27,0 & 14,0 \\
\hline $\mathrm{P}$ & $\mathrm{mg} . . \mathrm{dm}^{-3}$ & 0,6 & 0,4 \\
\hline $\mathrm{pH}$ & $\mathrm{H}_{2} \mathrm{O}$ & 5,1 & 5,3 \\
\hline $\mathrm{H}+\mathrm{Al}$ & cmol..dm ${ }^{-3}$ & 5,0 & 4,0 \\
\hline SB & cmol..dm ${ }^{-3}$ & 2,2 & 0,9 \\
\hline $\mathrm{t}$ & cmol..dm ${ }^{-3}$ & 2,6 & 1,3 \\
\hline $\mathrm{T}$ & cmol..dm ${ }^{-3}$ & 7,2 & 4,9 \\
\hline $\mathrm{m}$ & $(\%)$ & 16,0 & 30,0 \\
\hline V & $(\%)$ & 30,3 & 19,0 \\
\hline M.O & dag. $\mathrm{kg}^{-1}$ & 2,5 & 2,0 \\
\hline $\mathrm{Zn}$ & $\mathrm{mg} . . \mathrm{dm}^{-3}$ & 0,1 & 0,1 \\
\hline $\mathrm{Fe}$ & $\mathrm{mg} . . \mathrm{dm}^{-3}$ & 91,1 & 74,2 \\
\hline $\mathrm{Mn}$ & $\mathrm{mg} . . \mathrm{dm}^{-3}$ & 21,4 & 6,4 \\
\hline $\mathrm{Cu}$ & $\mathrm{mg} . . \mathrm{dm}^{-3}$ & 3,2 & 1,8 \\
\hline B & $\mathrm{mg} . . \mathrm{dm}^{-3}$ & 0,2 & 0,2 \\
\hline S & $\mathrm{mg} . . \mathrm{dm}^{-3}$ & 14,3 & 7,1 \\
\hline
\end{tabular}

Análises realizadas pelo Laboratório de Fertilidade do Solo do Departamento de Ciência do Solo da Universidade Federal de Lavras - UFLA. P e K: extrator Mehlich - 1; $\mathrm{Ca}, \mathrm{Mg}$ e Al: extrator $\mathrm{KCl} 1 \mathrm{~N} ; \mathrm{H}+\mathrm{Al}$ extraídos com acetato de cálcio $1 \mathrm{~N}$, $\mathrm{pH} 7,0$.

$\mathrm{O}$ experimento foi instalado num esquema fatorial $3 \times 3+1$, sendo utilizadas três fontes de silicato (Silifértil, Extrativa e Agronelli) e três doses (150, 300 e $450 \mathrm{~kg} / \mathrm{ha}$ ), mais um tratamento adicional, sem silicato(controle), totalizando 10 tratamentos. Foi utilizado o delineamento em blocos casualizados com quatro repetições. As composições químicas das três fontes são apresentadas na Tabela 2.

Tabela 2 - Porcentagem de $\mathrm{SiO}_{2}, \mathrm{CaO}, \mathrm{MgO}$ das fontes de silicato utilizadas.

\begin{tabular}{lccc}
\hline & $\mathrm{SiO}_{2}$ & $\mathrm{CaO}$ & $\mathrm{MgO}$ \\
\cline { 2 - 4 } Fonte & \multicolumn{3}{c}{-------\%------ } \\
\hline Silifértil & 12 & 41 & 7,3 \\
Agronelli & 23 & 41 & 11 \\
Extrativa & 25 & 23 & 0,55 \\
\hline
\end{tabular}


As parcelas experimentais foram constituídas por seis linhas de cana, espaçadas de $1,4 \mathrm{~m}$, com comprimento de 12 metros com área total de $100,8 \mathrm{~m}^{2}$. Como área útil da parcela $67,2 \mathrm{~m}^{2}$ consideram-se 4 linhas centrais. As parcelas foram espaçadas de 1 metro entre si, a fim de facilitar as avaliações e a visualização das estacas de identificação dos tratamentos.

Após o preparo convencional do solo, com aração e duas gradagens, realizou-se em novembro de 2003 o plantio da cana-de-açúcar (cana de ano).

O sulcamento foi realizado mecanicamente, na profundidade de $20-30 \mathrm{~cm}$, com sulcador de 2 linhas, espaçamento de $1,4 \mathrm{~m}$, plantio em fila dupla, média de 14 gemas por metro.

Não se fez a aplicação de calcário na área, para não ocorrerem possíveis interferências com as fontes e doses de silicato utilizadas.

Todas as parcelas receberam adubação química, baseada na análise de solo (CFSEMG, 1999), sendo aplicados nos sulco de plantio na forma de cloreto de potássio e superfosfato simples respectivamente, as quantidades de $120 \mathrm{~kg}$ de $\mathrm{K}_{\mathrm{g}}$. ha ${ }^{-1}$ de $\mathrm{K}_{2} \mathrm{O}$ e $120 \mathrm{~kg} \cdot$ ha $^{-1}$ de $\mathrm{P}_{2} \mathrm{O}_{5}$. A adubação de cobertura nitrogenada foi realizada aos 60 dias após o plantio com $40 \mathrm{~kg}$. ha-1 de $\mathrm{N}$ na forma de uréia, sendo o fertilizante aplicado nas entrelinhas da cultura.

Não houve necessidade de nenhum tipo de controle de pragas e doenças durante a condução do experimento, sendo feito apenas o controle de plantas daninhas através de capinas realizadas manualmente sempre que necessário. A colheita foi realizada de forma manual, sem queima prévia do canavial, em novembro de 2004 (ciclo de um ano).

A cultivar de cana-de-açúcar utilizada foi a SP 801816, que apresenta as seguintes características: bom perfilhamento e bom fechamento de entrelinhas; alta produtividade agrícola; não exigente em solos; maturação precoce; muito rica em sacarose; baixo teor de fibra; tombamento ausente; florescimento e chochamento ausentes, segundo Fernandes (1991).

O rendimento de colmos foi obtido através de pesagens realizadas em balança tipo dinamômetro, com capacidade para $30 \mathrm{~kg}$, seguindo metodologia de Mariotti \& Lascano (1969), citados por Arizono et al. (1998). Posteriormente, realizou-se a transformação do peso por parcela para toneladas por hectare.

Por ocasião da colheita foram retirados, aleatoriamente, 12 colmos da área útil de cada parcela, eliminando-se o palmito e a palha. Após a identificação, os colmos foram enviados para o laboratório da Usina Coinbra-Luciânia, em Lagoa da Prata, Minas Gerais, determinando-se a Fibra (\%) cana, Pol (\%) cana, Brix (\%) cana, ATR (kg de açúcar . $\mathrm{t}$ colmos $^{-1}$ ) e pureza (\%) cana.
Os dados foram submetidos à análise de variância, com realização do teste de $\mathrm{F}$, sendo as comparações entre os tratamentos integrantes do esquema fatorial efetuada através de análises de regressão. De acordo com recomendação de Yassin et al. (2002), as comparações dos tratamentos fatoriais com o controle foram feitas utilizandose o teste de Dunnett ao nível de 5\% de probabilidade.

\section{RESULTADOS E DISCUSSÃO}

Para rendimento de colmos (t.há $\left.{ }^{-1}\right)$ não ocorreram efeitos significativos de fontes, doses, interação fonte $\mathrm{x}$ dose, nem quando se compara o controle com a média do componentes do fatorial. Resultados semelhantes foram encontrados por Vitti et al. (1999), quando estudaram o efeito de diferentes fontes e doses de silício na cultura da cana-de-açúcar.

Os valores médios obtidos para rendimentos de colmos em função dos tratamentos de aplicados, são apresentados na tabela 3. Estes rendimentos são aqueles normalmente obtidos para cana de ano na região.

Tabela 3 - Rendimento de colmos ( $\mathrm{t}$. ha-1) em função das fontes e doses de silicato de cálcio aplicadas. UFLA, Lavras, MG, 2004.

\begin{tabular}{ccc}
\hline \multicolumn{2}{c}{ Tratamentos } & $\begin{array}{c}\text { Rendimento de } \\
\text { colmos }\left(\mathrm{t} \cdot \mathrm{ha}^{-1}\right)\end{array}$ \\
\cline { 1 - 2 } Fonte & Dose $\left({\left.\mathrm{Kg} . \mathrm{ha}^{-1}\right)}^{-1}\right.$ & 83,5 \\
Silifértil & 150 & 79,0 \\
Silifértil & 300 & 82,0 \\
Silifértil & 450 & 82,7 \\
Extrativa & 150 & 83,2 \\
Extrativa & 300 & 86,3 \\
Extrativa & 450 & 84,7 \\
Agronelli & 150 & 80,7 \\
Agronelli & 300 & 85,2 \\
Agronelli & 450 & 83,0 \\
\hline \multicolumn{2}{c}{ Média dos Fatoriais } & 93,5 \\
\hline \multicolumn{2}{l}{ Controle } &
\end{tabular}

O rendimentos médios de colmos obtidos no presente trabalho, encontram-se na faixa esperada para cana de ano (ciclo de 12 meses) na região sul de Minas Gerais, que varia de 80 a $100 \mathrm{t}$ de colmos por hectare.

Bittencourt et al. (2003), estudando os efeitos do silicato de cálcio em um Latossolo Vemelho Escuro encontraram aumentos na produção de colmos de canade-açucar, discordando dos resultados encontrados neste trabalho. No Havaí, a aplicação de silicatos (escórias de siderurgia) em Latossolos tem incrementado a produção 
de cana-de-açúcar em até 12 t.ha-1 ${ }^{-1}$ quando o teor de silício é bastante reduzido (FOX et al., 1967; PRADO, 2000). Gurgel (1979) mostrou que a aplicação de 3 t.ha ${ }^{-1}$ de silicato de cálcio puro, no plantio da cana-de-açúcar (CB 41-76), proporcionou aumento de $6,4 \%$ e $16 \%$ na produtividade de colmos de primeiro e segundo cortes, respectivamente. $\mathrm{O}$ uso de diferentes fontes doses tipos de solo e variedades podem explicar as divergências de resultados.

Não ocorreram efeitos significativos de fontes, doses e da interação fontes $\mathrm{x}$ doses para nenhuma das características químico-tecnológicas estudadas. Apenas quando se compara o controle com a média dos componentes do fatorial, encontram-se diferenças siginificativas para fibra $(\%)$ cana.

Observa-se, pela Tabela 4, que a média dos tratamentos que receberam aplicação de silicato de cálcio (componentes do fatorial) foi superior à média obtida para o controle (testemunha, sem aplicação de silicato). Onde se aplicou o silicato, o valor obtido para fibra (\%) cana foi de $11,70 \%$ contra $11,00 \%$ da testemunha. Isto pode ser explicado pelo fato de que o silício deposita-se nas plantas entre a cutícula e a epiderme, proporcionando maior rigidez (EPSTEIN, 1994).

Tabela 4 - Características químico-tecnológicas de canade-açúcar e açúcar total recuperável (ATR) em função das fontes e doses de silicatos de cálcio aplicadas UFLA, Lavras, MG, 2004.

\begin{tabular}{|c|c|c|c|c|c|c|}
\hline \multicolumn{2}{|c|}{ Tratamentos } & \multirow[b]{2}{*}{$\begin{array}{c}\text { Brix } \\
\% \text { cana }\end{array}$} & \multirow[b]{2}{*}{$\begin{array}{l}\text { Fibra } \\
\% \text { cana }\end{array}$} & \multirow[b]{2}{*}{$\begin{array}{c}\text { Pol } \\
\% \text { cana }\end{array}$} & \multirow[b]{2}{*}{$\begin{array}{c}\text { PZA } \\
\% \text { cana }\end{array}$} & \multirow[b]{2}{*}{$\begin{array}{l}\text { ATR } \\
(\mathrm{kg} / \mathrm{t})\end{array}$} \\
\hline Fonte & $\begin{array}{c}\text { Dose } \\
\left(\mathrm{Kg} \mathrm{ha}^{-1}\right)\end{array}$ & & & & & \\
\hline Silifértil & 150 & 20,1 & 11,8 & 15,4 & 87,1 & 147,1 \\
\hline Silifértil & 300 & 19,2 & 11,5 & 14,7 & 86,6 & 140,0 \\
\hline Silifértil & 450 & 19,9 & 11,6 & 15,3 & 87,2 & 145,9 \\
\hline Extrativa & 150 & 19,7 & 11,7 & 15,0 & 86,5 & 143,6 \\
\hline Extrativa & 300 & 19,6 & 11,6 & 15,0 & 86,5 & 143,0 \\
\hline Extrativa & 450 & 20,3 & 11,5 & 15,7 & 87,2 & 149,4 \\
\hline Agronelli & 150 & 19,6 & 11,6 & 14,9 & 86,4 & 142,6 \\
\hline Agronelli & 300 & 19,7 & 12,0 & 14,9 & 83,0 & 142,6 \\
\hline Agronelli & 450 & 19,8 & 11,8 & 15,1 & 86,5 & 144,0 \\
\hline \multicolumn{2}{|c|}{ Média dos Fatoriais } & 19,8 & $11,7 \mathrm{a}$ & 15,1 & 86,3 & 144,4 \\
\hline \multicolumn{2}{|l|}{ Controle } & 19,2 & $11,0 \mathrm{~b}$ & 14,8 & 86,9 & 141,0 \\
\hline \multicolumn{2}{|c|}{ C.V. $(\%)$} & 3,40 & 5,83 & 3,82 & 0,85 & 4,85 \\
\hline
\end{tabular}

* significativo em relação ao controle, ao nível de 5\% de probabilidade.
Os valores médios obtidos para todas as características químico-tecnológicas estudadas, em função dos tratamentos aplicados, são apresentados na tabela 4 . Estes valores encontrados estão dentro dos padrões aceitos para caracterizar uma cana madura (STUPIELLO, 1987).

Deve ser ressaltado que o pagamento de cana atual baseia-se no açúcar total recuperável (ATR), elemento importantíssimo para fornecedores de cana-de-açúcar destinada a indústria. Esta característica não foi afetada pela aplicação das diferentes fontes e doses de silicato de cálcio, sendo que na média dos componentes do fatorial, que receberam silicato, o ATR encontrado foi de 144,4 kg/t contra $141,0 \mathrm{~kg} / \mathrm{t}$ do controle, diferença não significativa (Tabela 4).

\section{CONCLUSÕES}

A aplicação de silicato de cálcio no sulco de plantio, independente de fontes e doses utilizadas, proporcionou um aumento no teor de fibra (\%) cana, mas não influenciou o rendimento de colmos e de açúcar total recuperável, assim como os teores de Brix, pol e pureza (\%).

\section{REFERÊNCIAS BIBLIOGRÁFICAS}

AGRIANUAL. Anuário da agricultura brasileira. São Paulo: FNP Consultoria e Agroinformativo, 2005. 520 p.

ANDERSON, D. L.; JONES, D. B.; SNYDER, G. H. Response of a rice and sugar cane rolation to calcium silicate slag on Everglabes Histossols. Agronomy Journal, Madison, v. 79, p. 531-535, 1987.

ARIZONO, H.; MATSUOKA, S.; GHELLER, Y.; HOFFMANN, H. P.; BASSICHELLO, A. I.; MENEZES, L. L. Alternativas para avaliação de produção de cana-deaçúcar. STAB, Piracicaba, v. 16, n. 5, p. 20, maio/jun. 1998.

BITTENCOURT, M. F.; NOKAGHI, R. M.; KORNDORFER, G. H.; VOSS, L. V.; JARUSSI, J. R.; CAMARGO, M. S.; PEREIRA, H. S. Efeito do silicato de cálcio sobre a produção e qualidade da cana-de-açúcar: usina Equipav. In: CONGRESSO BRASILEIRO DE CIÊNCIA DO SOLO, 29., 2003. Anais... [S.1.: s.n.], 2003. p. 66.

BRASIL. Ministério da Agricultura e Reforma Agrária. Normas climatológicas: 1961-1990. Brasília, DF: SSPI/ Embrapa, 1992. 84 p.

CHEONG, Y. W. Y.; HEITZ, A.; DEVILLE, J. The effect of silicion on sugar cane growth in pure nutrient solution. Jounal of the Science of Food and Agriculture, London, v. 24, n. 1, p. 113-115, 1973. 
COMISSÃO DE FERTILIDADE DO SOLO DO ESTADO DE MINAS GERAIS. Recomendações para o uso de corretivos e fertilizantes em Minas Gerais: $5^{\mathrm{a}}$ aproximação. Viçosa, 1999. 359 p.

DATNOFF, L. E.; SNYDER, G. H.; KORNDÖRFER, G. H Silicon in agriculture: studies in plant science. Amsterdam: Elsevier, 2001. 403 p.

EPSTEIN, E. The anomaly of silicon in plant biology. Proceedings Natl. Academy Science, Washington, v. 91, p. 11-17, 1994.

FARIA, R. J. Influência do silicato de cálcio na tolerância do arroz de sequeiro ao déficit hídrico do solo. 2000. $47 \mathrm{f}$. Dissertação (Mestrado) - Universidade Federal de Viçosa, Viçosa, 2000.

FERNANDES, A. C. Terceira geração de variedades de cana-de-açucar. São Paulo: Copersucar, 1991. 27 p. (Boletim técnico).

FOX, R. L.; SILVA, J. A.; YOUNGUE, O. R.; PLUCNETT, D. L.; SHERMAN, G. D. Soil and plants silicion and silicate response by sugar cane. Soil Science Society America Proceding, Madison, v. 31, p. 775-779, 1967.

GURGEL, M. N. A. Efeitos do silicato de cálcio e sua interação com o fósforo no estado nutricional, produtividade e qualidade tecnológica da cana-de-açúcar. 1979. 62 f. Dissertação (Mestrado em Solos e Nutrição de Plantas) - Escola Superior de Agricultura "Luiz de Queiroz", Universidade de São Paulo, Piracicaba, 1979.

KIDDER, G.; GASCHO, G. J. Silicate slag recommended for specified conditions in Florida sugarcane. Florida: University of Florida, 1977.

KORNDÖRFER, G. H. Adubação com Silício: uma alternativa para o aumento da produtividade e controle de doenças e pragas do arroz, milho e cana-de-açúcar. Uberlândia: UFU, 1996.

KORNDÖRFER, G. H.; PEREIRA, H. S.; CAMARGO, M. S. de. Silicato de cálcio e magnésio na agricultura. Uberlândia: UFU/ICIAG, 2002. (GPSi-ICIAG-UFU. Boletim técnico, 1).
MANECHINI, C.; DONZELLI, J. L. Recomendação da adubação e calagem com base na análise e características dos solos: princípios e aplicações. Piracicaba: Copersucar, 1984.

PRADO, R. M. Resposta da cana-de-açúcar à aplicação de escória silicatada como corretivo de acidez do solo. 2000. 79 f. Dissertação (Mestrado em Agronomia: Sistemas de Produção) - Faculdade de Engenharia da Universidade Estadual Paulista, Ilha Solteira, 2000.

PRADO, R. M.; FERNANDES, F. M. Resposta da cultura da cana-de-açúcar à aplicação de escória de siderurgia como corretivo de acidez do solo. Revista Brasileira de Ciência do Solo, Campinas, v. 25, p. 201-209, 2001.

QUAGGIO, J. A. Acidez e calagem em solos tropicais. Campinas: Instituto Agronômico, 2000. 111 p.

SILVEIRA JÚNIOR, E. G.; PENATTI, C.; KORNDÖRFER, G. H.; CAMARGO, M. S. de. Silicato de cálcio e calcário na produção e qualidade da cana-de-açúcar: usina Catanduva. In: CONGRESSO BRASILEIRO DE CIÊNCIAS DO SOLO:ALICERCE DOS SISTEMAS DE PRODUÇÃO, 39., 2003, Ribeirão Preto, SP. Resumos... Ribeirão Preto: USP, 2003. p. 66.

STUPIELlO, J. P. Cana como matéria prima. In: PARANHOS, S. B. (Coord.). Cana-de-açúcar: cultivo e utilização. Campinas: Cargill, 1987. v. 2, p. 759-804.

VITTI, G. C.; DEMATTÊ, J. L. I.; ROLIM, J. C.; FURLAN, L. R. Avaliação de diferentes fontes e doses de silício na cultura da cana-de-açúcar, em relação à produtividade de colmos e rendimento pol: relatório final. Piracicaba: ESALQ/USP, 1999. 39 p.

YASSIN, N.; MORAIS, A. R. de; MUNIZ, J. A. Análise de variância em um experimento fatorial de dois fatores com tratamentos adicionais. Ciência e Agrotecnologia, Lavras, p. 1541-1547, dez. 2002. Edição especial.

YOSHIDA, S. Chemical aspects of the role of silicon in physiology of the rice plant. Bulletin National Institute Agronomic Science, Tokio, v. 15, p. 1-58, 1965. 